\title{
Mechanism of cellulase action in textile processes
}

\author{
Artur Cavaco-Paulo \\ Department of Textile Engineering, University of Minho, 4800 Guimarães, Portugal
}

Received 3 December 1997

\begin{abstract}
The effects on fabric caused by cellulase enzymes always result from a process in which strong mechanical action affecting the fabric is provided. This paper discusses the interaction between cellulase activities and mechanical agitation during textile processes. Possible mechanisms are suggested for depilling and ageing effects accounting for the presence of mechanical factors. The paper includes a review of the mechanisms of indigo backstaining during cellulase washing. (C) 1998 Elsevier Science Ltd. All rights reserved.
\end{abstract}

Keywords: Cellulases; Textile finishing; Cellulosic fibres

\section{Introduction}

Cellulases are increasingly being used in the textile industry. Their most successful application is in producing the stone-washed look of denim garments (Cavaco-Paulo and Almeida, 1994; Cavaco-Paulo et al., 1996a; 1996b; 1997; 1998a; 1998b). Other processes that improve fabric appearance by removing fuzz fibres and pills or deliver softening benefits have also been introduced (Cavaco-Paulo and Almeida, 1994; Cavaco-Paulo et al., 1996a; 1996b; 1997; 1998a; 1998b). Increased use is also being made of cellulases in domestic fabric washing products where they are claimed to aid detergency and to clean fibre surfaces, improving appearance and colour brightness (CavacoPaulo, 1997). Nowadays, these finishing and washing effects represent the largest market for cellulase enzymes worldwide.

Cellulases are a multicomponent enzyme system, with endoglucanases (EGs) that hydrolyse cellulose chains randomly, cellobiohydrolases (CBHs) that hydrolyse cellobiose from the polymer ends and cellobiases that hydrolyse cellobiose to glucose. All these enzyme components act in a synergistic fashion during the degradation of cellulose. However, the slow kinetics of enzymatic degradation of crystalline cellulose (like cotton cellulose) allow fabric and fibre properties to be improved without excessive damage.

The finishing and washing effects delivered by cellulases are always applied in processes (rotating drum washers and jets) where strong mechanical action on the fabric is provided (Cavaco-Paulo et al., 1996a; 1996b; 1997; 1998a).
The level of mechanical agitation is known to increase fabric weight loss and to affect differently the relative activities of EG and $\mathrm{CBH}$ in a total crude mixture (Cavaco-Paulo et al., 1996b). This is a key issue for understanding the mechanisms by which the effects delivered by cellulases are obtained (Cavaco-Paulo et al., 1996a; 1996b; 1997; 1998a).

\section{Cellulase activities under zero level of mechanical agitation}

Available commercial cellulases for textiles are mainly produced from the fungi Humicola insolens (maximal activity at $\mathrm{pH}$ 7) and Trichoderma reesei (maximal activity at $\mathrm{pH}$ 5). Both crude enzymes from these fungi produce two cellobiohydrolases (CBH I and $\mathrm{CBH}$ II), several endoglucanases (EG I, EG II and other minor EGs) and at least one cellobiase. In Table 1, the activities of genetically engineered crudes and purified EG I and $\mathrm{CBH}$ I from $T$. reesei (measured without any mechanical agitation in a beaker standing in an incubator) towards cotton are displayed.

Elimination of EG I and EG II in the crude mixture produced from $T$. reese $i$ seems not to affect their ability to fully hydrolyse cotton cellulose, whereas the elimination of $\mathrm{CBH}$ I and $\mathrm{CBH}$ II limits the cotton solubilization to a value of just $3 \%$. Therefore the presence of $\mathrm{CBH}$ components in a cellulase mixture is essential for the complete hydrolysis of crystalline cellulose. However, $\mathrm{CBH}$ I alone seems to hydrolyse cotton very slowly (Table 1). Other studies on the distribution of DP on bacterial microcrystalline cellulose 
Table 1

Measured cellulase activities towards scoured cotton fabrics (adapted from Cavaco-Paulo et al. (1995; 1996c)

\begin{tabular}{|c|c|c|}
\hline Enzyme & Solubilization & Reducing sugars (as glucose) \\
\hline Total crude mixture (T. reesei) & $100 \%$ (13 days) & - \\
\hline Engineered crude ( $T$. reesei) ( $\mathrm{CBH}$ I and II eliminated or crude $-\mathrm{CBH}$ I and II) & $3 \%$ (20 days) & - \\
\hline Engineered crude (T. reesei) (EG I and II eliminated or crude - EG I and II) & $100 \%$ (14 days) & - \\
\hline EG I $(T$. reesei $)+\beta$-glucosidase & $1 \%$ (20 days) & $50 \mathrm{mg} \mathrm{l}^{-1}(1 \mathrm{~h})$ \\
\hline $\mathrm{CBH} \mathrm{I}($ T. reesei $)+\beta$-glucosidase & $7 \%$ (20 days) & $28 \mathrm{mg} \mathrm{l}^{-1}(1 \mathrm{~h})$ \\
\hline
\end{tabular}

Treatment conditions (crude mixtures): $35 \mathrm{mg} / \mathrm{g}$ protein/fabric, $\mathrm{pH} 5,50^{\circ} \mathrm{C}$.

Treatment conditions (purified enzymes): $3 \mathrm{mg} / \mathrm{g}$ cellulase/fabric, $10 \mathrm{mg} / \mathrm{g} \beta$-glucosidase/fabric, $\mathrm{pH} 5,50^{\circ} \mathrm{C}(\beta$-glucosidase from almonds, Sigma).

showed that neither CBH decreased DP even after longer hydrolysis times whereas EG I and EG II significantly decreased DP (Srisodsuk, 1994). These results suggest that $\mathrm{CBH}$ enzymes hydrolyse cellulose chains in a stepwise manner, chain by chain. Minor EG components seem to cooperate with the CBHs, in a synergistic fashion, to fully hydrolyse cotton (Table 1-activity of the CBH-rich enzyme). Despite the reduction of DP (Cavaco-Paulo et al., 1996c; Srisodsuk, 1994) by EGs, they seem to be unable to hydrolyse cotton alone. EG I is a faster enzyme than CBH I in the initial stages of hydrolysis (Table 1), but after the cleavage of the more accessible cellulose chains on the surface of the cotton fibres their action seems to end.

\section{The role of mechanical action during cellulase processing}

EG and $\mathrm{CBH}$ components seem to limit their action to the external accessible cellulose chains and not to diffuse inside the fibres. This is a very important issue since all effects delivered by cellulase enzymes are due to the surface modification of cellulosic fibres. However, strong mechanical action on the fabric is also provided during cellulase treatment in machines such as jets, rotating drum washers and winches. Therefore the activities of cellulase enzymes should also be studied at different levels of mechanical agitation. Zero level of mechanical action can be achieved by incubating the samples in a beaker at $20^{\circ} \mathrm{C}$ for a few days, simulating an impregnation process and incubation at room temperature (Pad-Batch). Low to higher levels of mechanical agitation can be achieved by incubating the pots (rotating vertically) in a water bath with 0 (low level of mechanical agitation) to 10-15 (high level of mechanical agitation) steel discs or balls inside the pots in a Linitesttype machine (Cavaco-Paulo, 1997; Cavaco-Paulo and Almeida, 1994; Cavaco-Paulo et al., 1996a; 1996b; 1996c; 1997; 1998a; 1998b).

The variation in the reducing end groups (cotton reducing power) in the cotton fibres can give important indications about the relative activities of EG and $\mathrm{CBH}$ in a cellulase crude mixture at two different levels of the mechanical agitation process. In fact, higher levels of mechanical agitation enhance the endoglucanase activity (Cavaco-Paulo et al., 1996b). Looking at the curve in Fig. 1 with no discs, the reducing end groups in the fibres increase to a maximum value and then decline with the total cellulase treatment time, whereas at higher levels of mechanical action a consistent increase is found. At lower levels of mechanical agitation the initial increase is caused by endowise action while the decrease is caused by exoaction, whereas at higher levels the mechanical action creates more sites for EG attack, consistently increasing the reducing ends on the fibre surface. These results suggest that mechanical agitation increases EG activity relative to $\mathrm{CBH}$ activity in a total crude mixture (Cavaco-Paulo et al., 1996b).

The interaction between cellulase action and mechanical agitation was also studied by separating these two key features in two sequential steps (Cavaco-Paulo et al., 1997). A fabric was enzymatically treated in a simulated Pad-Batch process with no agitation and rinsed with a strong alkali to desorb all bound enzyme. The fabric was dried and then washed in a process with strong mechanical agitation. The washing process yielded significant weight loss and the content of cotton reducing power (insoluble reducing ends) was reduced. This seems to indicate that mechanical action will preferentially remove microfibrillar material with a high content of reducing ends.

Further understanding of these phenomena can be gained from the adsorption studies shown in Fig. 2. The increased mechanical action by a number of discs will increase the saturation levels for the cellulase mixtures, specially for the EG-rich cellulase. However, the increase of saturation levels by higher levels of mechanical agitation will lead to a decrease of cellulase adsorption. Further evidence to explain this increased desorption phenomenon is provided by the fact that high mechanical action levels will cause

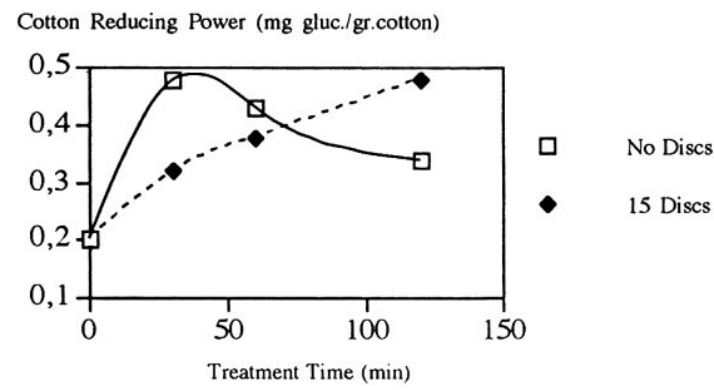

Fig. 1. Cotton reducing power versus treatment time of samples treated with a total cellulase crude mixture (T. reesei) in Linitest pots (adapted from Cavaco-Paulo et al. (1996b)). 


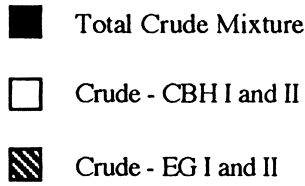

Saturation Levels (mg enzyme/g cotton) Adsorption Constant $(\mathrm{ml} / \mathrm{mg})$
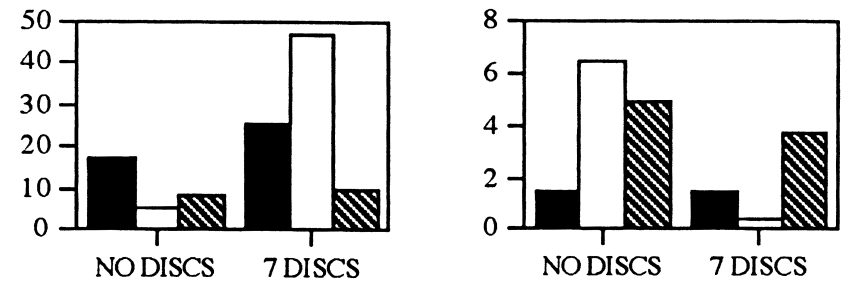

Fig. 2. Saturation levels and adsorption constant measured during simulated cellulase (T. reesei) process in a Linitest machine (adapted from Cavaco-Paulo et al. (1998a)).

insoluble weight loss, i.e. cotton powder. This cotton powder has a higher surface area, increasing desorption of the enzyme from the fabric. Measurement of the levels of protein bound to cotton indicate that they are 6-7 times higher in the cotton powder than in the fabric.

Previous observations (Fig. 1) for interaction between mechanical agitation and enzyme activities can be confirmed by the results (Fig. 3) for EG- and $\mathrm{CBH}$-rich crude mixtures. The relative activity of the EG-rich crude is increased whereas that of the $\mathrm{CBH}$-rich crude is decreased when the process with a high level of mechanical agitation (HLMA) is compared with the Pad-Batch process with no agitation.

The high interaction between EG activity (EG-rich crude) and mechanical agitation could completely break a piece of fabric into a microfibrillar mass whereas the $\mathrm{CBH}$-rich crude will leave the main fabric structure intact under similar treatment conditions. These treatment conditions are aggressive (in $500 \mathrm{ml}$ Linitest pots with 15 discs, $10 \%$ enzyme to the fabric and $100 \mathrm{ml}$ of liquor) but the test gives a clear idea of the kind of interaction between mechanical agitation and enzyme activities.

Higher weight loss is found for flannelette in the HLMA process only, whereas a similar weight loss is found for both fabrics in the Pad-Batch process (Fig. 3). This is due to the removal of pills present at the surface of the flannelette fabric at high levels of mechanical agitation (poplin is a very tight structure fabric with no pills). In fact, a high load of microfribrillar material is present in the liquor of the HLMA process. The results of Fig. 4 show similar loss of strength for both fabrics for the Pad-Batch process with no agitation, whereas for the HLMA process the poplin shows significantly higher strength loss than the flannelette. This could mean that with zero agitation both fabrics (with and without pills) have a similar degradation mechanism, whereas at higher levels of mechanical agitation the degradation in the poplin is on the main fabric structure while the degradation in the flannelette is mainly on the raised pills. These results indicate that mechanical agitation will drive the action of cellulases towards degradation of the surface pills in a fabric and that this is an essential issue to obtain good depilling effects.

\section{Other features during cellulase processing}

It is shown that for all kinds of processes EG activity leads to higher levels of strength loss (Fig. 4). This is what could be expected from a classical endowise cleavage of the cellulose chains in the fibres. This is a very important result in the formulation of a cellulase product since a significant effect should be obtained without significant fabric damage and this can be achieved just by balancing EG activity in the mixture used.

Previous treatments such as cotton scouring and bleaching will increase the dry cellulose content of the fibre up to $99 \%$, and will increase the extent of cellulase hydrolysis (Cavaco-Paulo, 1997). Previous mercerization will also increase the extent of hydrolysis due to the larger available surface area of the more cylindrical mercerized fibres (Cavaco-Paulo et al., 1998a).

Most cellulase treatments in the industry take place after dyeing in the final stages of finishing. Therefore the effect of previous dyeing of cotton is a very important issue for cellulase processing.

Vat dyes are firmly trapped inside the fibres and seem not to interfere with the cellulase hydrolysis process (CavacoPaulo et al., 1998a). Direct dyes are large planar molecules fixed to the cellulose fibres by ionic and VW linkages; therefore the access of cellulase enzymes is greatly reduced and lower levels of hydrolysis are found on previously directdyed cotton (Cavaco-Paulo et al., 1998a). Reactive dyes are covalently linked to cellulose chains; therefore it is expected that enzymes with a tunnel-shaped active site will have strong inhibition on the reactive-dyed substrate; this is the case mainly for $\mathrm{CBH}$-rich enzymes. However, some inhibition is also found for EG-rich mixtures (Cavaco-Paulo et al., 1998a). Neither vat nor direct dyes were released into 


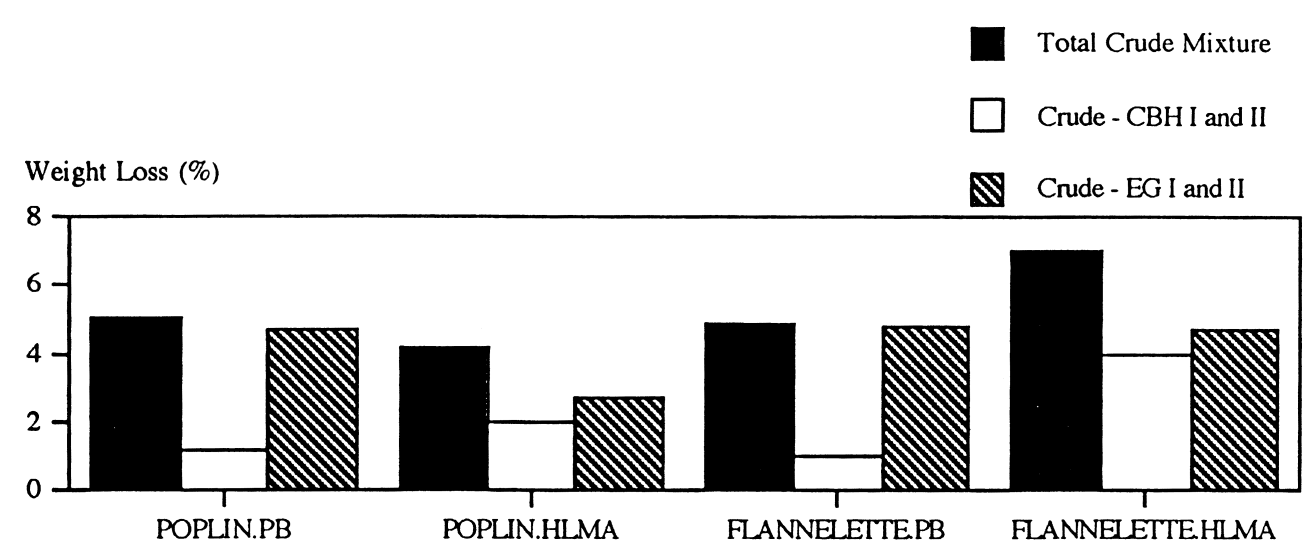

Fig. 3. Weight loss for various processes and fabrics. PB: simulated Pad-Batch process with zero level of mechanical agitation. HLMA: high level of mechanical agitation with 15 discs inside the Linitest pots (adapted from Cavaco-Paulo et al. (1996a)).

solution until a lower weight loss $(<5 \%)$ occurred, whereas the reactive dye came into solution attached to soluble sugars from the very beginning of hydrolysis. Therefore reactive-dyed cotton behaves like any other cellulose derivative where it is known to exhibit strong $\mathrm{CBH}$ inhibition.

Dyeability and moisture recovery are not expected to change after cellulase treatment, since no changes were found in the crystallinity of cellulase-treated cotton (Cavaco-Paulo et al., 1996b; 1996c). However, due to defibrillation, water retention has been shown to increase. Sometimes slightly deeper shades are apparently obtained after cellulase hydrolysis, due to the enzyme treatment cleaning fibre surfaces to give this effect (Cavaco-Paulo et al., 1996c).

\section{Cellulase process mechanisms}

On the basis of previous experimental evidence, the mechanisms for cooperation between cellulase activities and mechanical agitation could be:

1. EG randomly cleaves the accessible cellulose chains.
2. Mechanical action on the fibres will raise these broken ends, creating microfibrils and consequently more sites in the fibres for EG attack.

3. (Lack of CBHs) Since mechanical agitation desorbs EGs rapidly, the endowise action will proceed in other randomly accessible points of the fibre. A shorter process will result in a fibre surface with raised microfibrils and several microfibrils in the liquor, whereas a longer process will result in the complete transformation of the fibres and the fabric structure into a mass of microfibrillar material.

4. (Presence of $\mathrm{CBHs}$ ) $\mathrm{CBH}$ starts the hydrolysis on the more accessible raised broken chains, leaving localized under chains for attack by EGs. The CBH enzymes seem to focus the action of EG. The mechanical action will raise material from the holes formed, helping the enzymes in a possible 'cutting' process. When the fibres are weak, mechanical action will completely break them. However, more experimental evidence is needed to explain this mechanical cutting off of fibres and microfibrils.

In a cleaning/depilling mechanism the presence of both enzymes is essential; the mechanical action will drive the

Total Crude Mixture

Crude - CBH I and II

Ratio Strength Loss (\%)/Weight Loss (\%)

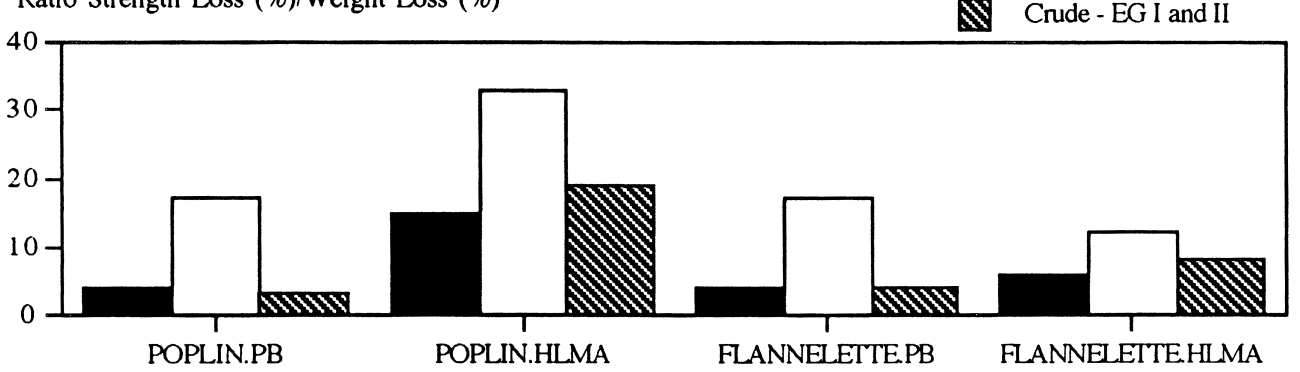

Fig. 4. Ratio of strength loss to weight loss for various processes and fabrics. PB: simulated Pad-Batch process with zero level of mechanical agitation. HLMA: high level of mechanical agitation with 15 discs inside the Linitest pots (adapted from Cavaco-Paulo et al. (1996a)). 
action of the enzymes towards the pills and loose fibres, cutting them as explained above.

The aged look results from a highly defibrillated fibre surface. If the fibre is dyed the aged fabric shows a low level of brightness, similarly to what is observed after many domestic washes. The ageing process with EGs or EG-rich cellulases and mechanical action will produce a similar effect at the fibre level.

The objective of ageing denim fabrics is the nonhomogeneous removal of dye from the fabric, giving the fashionable contrast of various shades of blue. Indigo dye is 'trapped' inside the fibres and any process that exposes these inner fibre layers, releasing the dye, will produce the required effect. Processes with high levels of mechanical friction with pumice stones will produce strong mechanical abrasion of fibre surfaces, releasing the indigo dye and producing the stone-wash effect. Cellulase enzymes (total crudes or EG-rich crudes) can produce the same effect with much less mechanical means. This is the main value of the enzymatic washing process.

\section{Indigo backstaining during enzymatic washing}

The backstaining of indigo dye during the washing of denim garments with cellulases seems to be a major problem. Experimental evidence indicates that Humicola insolens enzymes (neutral) give less dye repositioning than Trichoderma reesei enzymes (acid), suggesting that $\mathrm{pH}$ is the major factor in controlling backstaining (Cavaco-Paulo et al., 1998b). Recent studies indicate that the ability of cellulase enzymes to be strongly adsorbed on cotton substrates seems to be the main cause of backstaining (Cavaco-Paulo et al., 1998b). This confirms why some detergents that desorb cellulases reduce backstaining. Furthers experiment indicate that the nature of the enzyme used is an important issue, because indigo dye seems to stain better in Trichoderma than in Humicola enzymes (CavacoPaulo et al., 1998b). The first indication that the cellulase binding process plays a major role during backstaining was suggested by Genencor patents in which indigo staining levels were reduced by the use of proteases that prevented the enzyme binding during washing (Cavaco-Paulo et al., 1998b). The use of some cellulase components with low binding behaviour also showed low indigo staining levels (Cavaco-Paulo et al., 1998b).

\section{References}

Cavaco-Paulo, A. (1991). In: K.L. Eriksson \& A. Cavaco-Paulo (Eds.), Enzyme Applications for Fiber Processing, ACS Symposium Series pp. $180-189$.

Cavaco-Paulo, A., \& Almeida, L. (1994). Biocatalysis, 10, 353-360.

Cavaco-Paulo, A., Buchert, J., Siika-aho, M., Almeida, L., \& Viikari, L. (1995). In: PacifChem, Honolulu, HI, 17-22 December.

Cavaco-Paulo, A., Almeida, L., \& Bishop, D. (1996). Textile Chemist \& Colorist, 28 (6), 28-32.

Cavaco-Paulo, A., Almeida, L., \& Bishop, D. (1996). Textile Research Journal, 64, 287-294.

Cavaco-Paulo, A., Almeida, L., \& Bishop, D. (1996c). In: P.A. Williams \& J.F. Kennedy (Eds.), The chemistry and processing of wood and plant fibrous materials (pp. 227-234). Cambridge: Woodhead.

Cavaco-Paulo, A., Cortez, J., \& Almeida, L. (1997). Journal of the Society of Dyers and Colorists, 117, 17-21.

Cavaco-Paulo, A., Almeida, L., \& Bishop, D. (1998a). Textile Research Journal, 68(4), 273-280.

Cavaco-Paulo, A., Morgado, J., Almeida, L., \& Kilburn, D. (1998b). Textile Research Journal, 68(6), 398-401.

Srisodsuk, M. (1994). PhD thesis. Espoo, Finland: VTT Publications, Vol. 188. 